\title{
The complete set of minimal simple graphs that support unsatisfiable 2-CNFs
}

\author{
Vaibhav Karve ${ }^{\mathrm{a}}$, Anil N. Hirani ${ }^{\mathrm{a}, *}$ \\ ${ }^{a}$ Department of Mathematics, University of Illinois at Urbana-Champaign, \\ 1409 W. Green Street, Urbana, IL 61801
}

\begin{abstract}
A propositional logic sentence in conjunctive normal form that has clauses of length two (a 2-CNF) can be associated with a multigraph in which the vertices correspond to the variables and edges to clauses. We first show that every such sentence that has been reduced, that is, which is unchanged under application of certain tautologies, is equisatisfiable to a $2-\mathrm{CNF}$ whose associated multigraph is, in fact, a simple graph. Our main result is a complete characterization of graphs that can support unsatisfiable 2-CNF sentences. We show that a simple graph can support an unsatisfiable reduced 2-CNF sentence if and only if it contains any one of four specific small graphs as a topological minor. Equivalently, all reduced 2-CNF sentences supported on a given simple graph are satisfiable if and only if all subdivisions of those four graphs are forbidden as subgraphs of of the original graph. We conclude with a discussion of why the Robertson-Seymour graph minor theorem does not apply in our approach.
\end{abstract}

Keywords: boolean satisfiability, conjunctive normal form, propositional logic, graph minors, topological minors, edge contraction, subdivision

\section{Introduction}

Given a sentence in propositional logic, the satisfiability decision problem is to determine if there exists a truth assignment for the variables that makes the sentence true. Let $V$ be a finite set of Boolean variables, $\neg V$ be the set $\{\neg x: x \in V\}$ of negations, and let the symbols $T$ and $\perp$ be True and False values of variables. We define the set of literals obtained from $V$ to be the set $V \cup \neg V \cup\{\top, \perp\}$.

A Conjunctive Normal Form (CNF) on $V$ is a conjunction of one or more clauses, where each clause is a disjunction of literals. A CNF (or clause) is reduced if it is unchanged under application of all the tautologies listed below

$$
\begin{array}{llll}
x \vee \top=\top, & x \wedge \top=x, & x \vee x=x, & \neg(\neg x)=x, \\
x \vee \perp=x, & x \wedge \perp=\perp, & x \wedge x=x, & x \vee \neg x=\top .
\end{array}
$$

It is a fact that every CNF (or clause) is logically equivalent to a reduced CNF (or clause). The length of a reduced clause is the number of literals in the clause. For $k \in \mathbb{N}$, a reduced CNF is a reduced $k-C N F$ if all of its clauses have length $k$. Additionally, for ease of stating results, the CNFs $\top$ and $\perp$ will be treated as $k$-CNFs for every $k \in \mathbb{N}$. We will refer to the satisfiability decision problem as SAT and the satisfiability problem for $k$-CNFs as $k$-SAT.

Instead of algorithmic issues our aim in this paper is to study the structure of unsatisfiable 2-CNF sentences in a sense that will be made precise later. For completeness, here we briefly summarize the relevant fundamental algorithmic results for satisfiability problems. The 2-SAT problem is in P. An $\mathcal{O}\left(n^{4}\right)$

* Corresponding author 
algorithm was given by Krom [1] and a linear-time algorithm by Even, Itai and Shamir [2] and Aspvall, Plass and Tarjan [3]. All solutions of a given 2-CNF sentence can be listed efficiently using an algorithm by Feder [4].

In contrast with the algorithmic tractability of 2-SAT, the SAT problem in general is NP-complete as was shown by Cook and by Levin independently $[5,6]$. As part of the proof of the NP-completeness of SAT, they also proved that every logical sentence can be rewritten as a CNF while changing its length by no more than a constant factor. Schaefer's dichotomy theorem states necessary and sufficient conditions under which a finite set $S$ of relations over the Boolean domain yields polynomial-time or NP-complete problems when the relations of $S$ are used to constrain some of the propositional variables [7]. Thus [7] gives a necessary and sufficient condition for SAT-type problems to be in P vs. NP.

Our paper relates properties of certain graphs to satisfiability. An early connection between satisfiability and graphs was in the proof of NP-completeness of various graph problems, such as the clique decision problem and the vertex cover problem, by Karp [8]. One of the linear-time algorithms for 2-SAT [3] mentioned above also related graphs and satisfiability in its use of strongly-connected graph components as a tool for deciding satisfiability.

We explore the structures of unsatisfiable 2-CNFs by relating reduced 2-CNFs to graphs and examining which graphs can support unsatisfiable sentences. Given a 2-CNF, an associated multigraph can be created by identifying the variables as vertices and each clause as an edge. Since multiple clauses may involve the same two variables, it is not immediate that it is sufficient to consider graphs rather than multigraphs. That it indeed is so is the content of Section 4. In Section 5 we theorems about the relation between graphs that support unsatisfiable sentences. Theorem 4 in that section shows that the family of simple graphs that can support an unsatisfiable sentence is closed under graph homeomorphism while Theorem 5 shows that a graph can support an unsatisfiable sentence if one of its subgraph can. Theorem 7 shows that if a graph can support an unsatisfiable sentence, then the graphs obtained by edge-contractions at edges not contained in triangles can. Section 7 is about connectivity properties of graphs that we need to prove the main result. The main result of this paper is Theorem 20 in which we give a complete characterization of graphs that can support unsatisfiable sentences. This is given in the form of a finite set of obstructions to supporting only satisfiable sentences. In Section 9 we discuss how our approach differs from the application of finite obstructions (forbidden minors) theory developed in the Robertson-Seymour graph minor theorem published in a series of papers starting with [9] and ending with [10].

\section{Preliminaries}

For a reduced CNF $S$ and a variable $v$, we denote by $S[v:=\top]$ the reduced CNF obtained by

a) setting all occurrences of $v$ in $S$ to $\mathrm{T}$,

b) setting all occurrences of $\neg v$ in $S$ to $\perp$, and

c) reducing the CNF thus obtained, that is, applying all the tautologies listed in Section 1 to the CNF in order to obtain a reduced $\mathrm{CNF}$.

Similarly, the reduced CNF obtained by setting $v$ to $\perp$, setting $\neg v$ to $\top$, and then performing the tautological reductions is denoted by $S[v:=\perp]$. If we set multiple variables to true or false, we use the notation $S[\{a, b\}:=\top ;\{c, d\}:=\perp]$ as short-hand for $S[a:=\top][b:=\top][c:=\perp][d:=\perp]$ and so on for variables $a, b, c$ and $d$. Note that the order in which we set variables to true or false is not important, as it yields logically equivalent results.

We now define some more adjectives for CNFs. A reduced CNF $S$ is true if $S=\top$, false if $S=\perp$, and nontrivial if $S$ is neither true nor false. A reduced CNF $S$ is satisfiable if there is a subset $V_{1}$ of $V$ such that $S\left[V_{1}:=\top ; V-V_{1}:=\perp\right]$ is true. A reduced CNF is unsatisfiable if it is not satisfiable. Two reduced CNFs, $S$ and $S^{\prime}$ are equisatisfiable, denoted by $S \sim S^{\prime}$, if either both are satisfiable or both are unsatisfiable. 


\section{Graph associated with a reduced CNF}

We define an operation $|\cdot|: V \cup \neg V \rightarrow V$ as $|x|=|\neg x|=x$, for every $x \in V$. Let $\bigvee_{i=1}^{k} a_{i}$ be a nontrivial clause denoted by $\alpha$, where $k \in \mathbb{N}$ and $a_{i} \in V \cup \neg V$ for $i \in\{1, \ldots, k\}$. We extend the domain of the operation $|\cdot|$ to include clauses like $\alpha$ by defining $|\alpha|=\left\{\left|a_{i}\right|: i \in\{1, \ldots, k\}\right\}$.

Let $S$ be a nontrivial 2-CNF. We can write it as $S=\bigwedge_{j=1}^{n} \alpha_{j}$, where $n \in \mathbb{N}$ and each $\alpha_{j}$ is a clause of length 2. To $S$ we associate a multigraph $\mathcal{M G}(S)$ having vertex set $\bigcup_{j=1}^{n}\left|\alpha_{j}\right|$ and having an edge $\left|\alpha_{j}\right|$ for every $j \in\{1, \ldots, n\}$. Note that a variable $x$ and its negation $\neg x$ are both represented by a single vertex (also labeled $x$ ) in $\mathcal{M G}(S)$. The 2-CNF $S$ being nontrivial guarantees that $\mathcal{M G}(S)$ is a nonempty multigraph, thus avoiding pathological cases. The nontriviality of $S$ implies that $S$ is reduced, thus ensuring there are no self-loops in $\mathcal{M G}(S)$, since self-loops can only occur due to clauses $(x \vee \neg x)$ and $(x \vee x)$.

If a multigraph $G$ is the associated multigraph of a 2 -CNF $S$, then we say $S$ is supported on $G$. A nontrivial 2-CNF $S$ is simple if its associated graph is simple. In such a case, we drop the prefix "multi-" and denote the associated graph $\mathcal{M G}(S)$ simply by $\mathcal{G}(S)$. We denote by $\mathcal{U}$ the following family of graphs

$$
\mathcal{U}=\{\mathcal{G}(S): S \text { is an unsatisfiable simple 2-CNF }\} .
$$

Our aim in this paper is to characterize the elements of $\mathcal{U}$. First, we note that $\mathcal{U}$ is nonempty since

$$
S=(a \vee b) \wedge(\neg a \vee c) \wedge(\neg b \vee c) \wedge(\neg c \vee d) \wedge(\neg d \vee e) \wedge(\neg d \vee f) \wedge(\neg e \vee \neg f),
$$

is an unsatisfiable simple 2-CNF supported on the following graph.

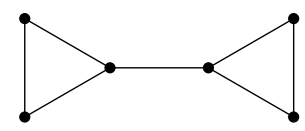

\section{Simple CNFs suffice}

In this section, we show that every nontrivial reduced 2-CNF is equisatisfiable to a simple 2-CNF. Thus when studying satisfiability of 2-CNFs we only need consider those that are simple.

First, we make the observation that for any $a \in V$, there are at most two length 1 clauses, namely $a$ and $\neg a$. Such clauses involving a single variable $a$ (or its negation) will be referred to as (a)-clauses. For every $a, b \in V$, there are at most four length 2 clauses, namely $(a \vee b),(a \vee \neg b),(\neg a \vee b)$ and $(\neg a \vee \neg b)$. Consequently, for any reduced 2-CNF $S$, edges in $\mathcal{M G}(S)$ have a maximum multiplicity of 4 . Such clauses involving both $a$ and $b$ (or their negations) will be referred to as $(a, b)$-clauses.

Lemma 1. Let $S$ and $R$ be reduced $C N F$ s. Let $a \in V$ such that $R$ does not contain any (a)-clauses.

a) If $S=R \wedge a \wedge \neg a$, then $S$ is unsatisfiable.

b) If $S=R \wedge a$, then $S \sim R[a:=T]$.

Proof. Let $S=R \wedge a \wedge \neg a$. We can simplify this as $S=R \wedge \perp=\perp$. Therefore, $S$ is unsatisfiable.

Let $S=R \wedge a$. Any truth assignment that satisfies $S$ must have $[a:=\top]$ and must therefore satisfy $R[a:=\top]$. Conversely, consider a truth assignment that satisfies $R[a:=\mathrm{T}]$. Recall that $R[a:=\mathrm{\top}]$ denotes the reduced CNF resulting from replacing $a$ with $\top$ and $\neg a$ with $\perp$ in $R$. Hence, the variable $a$ (or its negation) are not present in $R[a:=\top]$. As a result, any truth assignment that satisfies $R[a:=\top]$ must leave the variable $a$ unassigned. By additionally setting $[a:=\top]$, we obtain an assignment that satisfies $S$. We conclude that $S \sim R[a:=\top]$.

Lemma 2. Let $S$ and $R$ be reduced 2-CNFs. Let $a, b \in V$ such that $R$ does not contain any $(a, b)$-clauses.

a) If $S=R \wedge(a \vee b) \wedge(a \vee \neg b) \wedge(\neg a \vee b) \wedge(\neg a \vee \neg b)$, then $S$ is unsatisfiable.

b) If $S=R \wedge(a \vee b) \wedge(a \vee \neg b) \wedge(\neg a \vee b)$, then $S \sim R[\{a, b\}:=\top]$. 
c) If $S=R \wedge(a \vee b) \wedge(a \vee \neg b)$, then $S \sim R[a:=\top]$.

d) If $S=R \wedge(a \vee b) \wedge(\neg a \vee b)$, then $S \sim R[b:=\top]$.

e) If $S=R \wedge(a \vee b) \wedge(\neg a \vee \neg b)$, then $S \sim R[b:=\neg a]$

Proof. Suppose $S$ is of the form $R \wedge(a \vee b) \wedge(a \vee \neg b) \wedge(\neg a \vee b) \wedge(\neg a \vee \neg b)$. As a result of the logical implications $(a \vee b) \wedge(a \vee \neg b) \rightarrow a$ and

$(\neg a \vee b) \wedge(\neg a \vee \neg b) \rightarrow \neg a$, we can deduce the implication $S \rightarrow a \wedge \neg a=\perp$. Therefore, $S$ is unsatisfiable.

Let $S=R \wedge(a \vee b) \wedge(a \vee \neg b) \wedge(\neg a \vee b)$. The three $(a, b)$-clauses of $S$ imply $(a \wedge b)$. Hence any truth assignment that satisfies $S$ must also satisfy $(a \wedge b)$. We can conclude that such an assignment must have $[\{a, b\}:=\top]$. Hence this assignment must also satisfy $R[\{a, b\}:=\top]$. Thus, $S$ satisfiable implies $R[\{a, b\}:=\top]$ satisfiable. Conversely, consider a truth assignment that satisfies $R[\{a, b\}:=\top]$. By additionally setting $[\{a, b\}:=\top]$, we obtain an assignment that satisfies $S$. We conclude $S \sim R[\{a, b\}:=\top]$.

Let $S=R \wedge(a \vee b) \wedge(a \vee \neg b)$. Since $(a \vee b) \wedge(a \vee \neg b) \rightarrow a$, any truth assignment that satisfies $S$ must have $[a:=\top]$. Thus, such a truth assignment must also satisfy $R[a:=\top]$. Conversely, consider a truth assignment that satisfies $R[a:=\top]$. By additionally setting $[a:=\top]$, we obtain an assignment that satisfies $S$. We conclude that $S \sim R[a:=\top]$.

Let $S=R \wedge(a \vee b) \wedge(\neg a \vee b)$. Since $(a \vee b) \wedge(\neg a \vee b) \rightarrow b$, by a similar reasoning to the previous case, we conclude that $S \sim R[b:=\top]$.

Lastly, let $S=R \wedge(a \vee b) \wedge(\neg a \vee \neg b)$. Any truth assignment that satisfies $S$ must map $a$ and $b$ to opposite truth values in order to satisfy the two $(a, b)$-clauses. Thus, such a truth assignment must also satisfy $R[b:=\neg a]$. Conversely, consider a truth assignment that satisfies $R[b:=\neg a]$. In this truth assignment, we have no assignment for $b$ (as all occurrences of $b$ have been replaced by $\neg a$ ). By additionally setting $[b:=\neg a]$, we obtain an assignment that satisfies $S$. We conclude that $S \sim R[b:=\neg a]$.

Lemma 3. Let $S$ be a reduced CNF with clauses of length at most 2 .

a) If $S$ has an (a)-clause for some variable a, then there exists a reduced 2-CNF $S^{\prime}$ equisatisfiable to $S$.

b) If $S$ has four $(a, b)$-clauses for some $a, b \in V$, then $S$ is unsatisfiable.

c) If $S$ has three or fewer $(a, b)$-clauses for every $a, b \in V$, then there exists a 2-CNF $S^{\prime}$ equisatisfiable to $S$ such that either $S^{\prime}$ is trivial or $S^{\prime}$ is simple.

Proof. Let $S$ be a reduced CNF having four $(a, b)$-clauses for some $a, b \in V$. We can then write $S$ in the form

$$
S=R \wedge(a \vee b) \wedge(a \vee \neg b) \wedge(\neg a \vee b) \wedge(\neg a \vee \neg b),
$$

where $R$ is a reduced 2-CNF not containing any $(a, b)$-clauses. By Lemma 2 (a), we conclude that $S$ is unsatisfiable.

Let $S$ have an $(a)$-clause for some $a \in V$. The reduced CNF $S$ has either exactly one or exactly two (a)-clauses. If $S$ has exactly two $(a)$-clauses, then we can write $S=R \wedge a \wedge \neg a$, where $R$ is a reduced CNF not containing any $(a)$-clauses. By Lemma 1 (a), we conclude that $S$ is unsatisfiable. Hence we can write $S \sim S_{1}$, where $S_{1}$ is the trivially false 2 -CNF $\perp$.

If $S$ has exactly one (a)-clause, then by exchanging $\neg a$ with $a$ if needed, we can ensure that $S$ is of the form $R \wedge a$, where $R$ is a reduced CNF not containing any (a)-clauses. By Lemma 1 (b), we conclude that $S \sim R[a:=\top]$. The reduced CNF $R[a:=\top]$ has no $(a)$-clauses but might have either one or two (b)-clauses for some other variable $b$. By repeated application of Lemma 1 (a) and (b), we can find a resulting CNF $S^{\prime}$ equisatisfiable to $S$, and having no length 1 clauses. Since $S^{\prime}$ has no length 1 clauses, it is a 2 -CNF.

Having dealt with the case when $S$ has four $(a, b)$-clauses for some $a, b \in V$, we can assume throughout the remainder of the proof that $S$ has three or fewer $(a, b)$-clauses for every $a, b \in V$. Also, having dealt with the case when $S$ has an $(a)$-clause for some $a \in V$, we can henceforth assume that $S$ is a reduced 2-CNF.

Let $a, b \in V$ such that $S$ has exactly two $(a, b)$-clauses. By exchanging $a$ with $\neg a$ and/or $b$ with $\neg b$ we can ensure that one of the two $(a, b)$-clauses is $(a \vee b)$. Thus $S$ has one of three forms, which we label $S_{1}, S_{2}$ and $S_{3}$

$$
S_{1}=R \wedge(a \vee b) \wedge(a \vee \neg b), \quad S_{2}=R \wedge(a \vee b) \wedge(\neg a \vee b), \quad S_{3}=R \wedge(a \vee b) \wedge(\neg a \vee \neg b) ;
$$


where $R$ is a reduced 2-CNF not containing any $(a, b)$-clauses. Lemma 2 (c) implies that $S_{1} \sim R[a:=\top]$. We note that $R[a:=\top]$ is a reduced CNF with clauses of length either 2 or 1 . By repeated application of Lemma 1 (a) and (b), we can again find a reduced 2-CNF $R_{1}^{\prime}$ such that $R_{1}^{\prime} \sim R[a:=\mathrm{\top}] \sim S_{1}$, and such that $R_{1}^{\prime}$ (as opposed to $S_{1}$ ) has no $(a, b)$-clauses. Repeating this process for every $c, d \in V$ such that $R_{1}^{\prime}$ has exactly two $(c, d)$-clauses gives us a resulting 2-CNF $S_{1}^{\prime}$ equisatisfiable to $S_{1}$ such that $S_{1}^{\prime}$ has either three, or one, or zero $(a, b)$-clauses for every $a, b \in V$.

Lemma 2 (d) implies that $S_{2} \sim R[b:=\top]$. Repeatedly applying Lemma 1 (a) and (b), we can find a reduced 2-CNF $R_{2}^{\prime}$ such that $R_{2}^{\prime} \sim S_{2}$ and such that $R_{2}^{\prime}$ (as opposed to $S_{2}$ ) has no $(a, b)$-clauses. If $R_{2}^{\prime}$ has exactly two $(c, d)$-clauses for some $c, d \in V$, then repeating the above process gives us a resultant 2-CNF $S_{2}^{\prime}$ equisatisfiable to $S_{2}$ such that $S_{2}^{\prime}$ has either three, or one, or zero $(a, b)$-clauses for every $a, b \in V$.

Lemma 2 (e) implies that $S_{3} \sim R[b:=\neg a]$. By repeated application of Lemma 1 (a) and (b), we can again find an reduced 2-CNF $R_{3}^{\prime}$ such that $R_{3}^{\prime} \sim S_{3}$ and such that $R_{3}^{\prime}$ (as opposed to $S_{3}$ ) has no $(a, b)$-clauses. If $R_{3}^{\prime}$ has exactly two $(c, d)$-clauses for some $c, d \in V$, then repeating the above process gives us a resultant 2-CNF $S_{3}^{\prime}$ equisatisfiable to $S_{3}$ such that $S_{3}^{\prime}$ has either four, or three, or one, or zero $(a, b)$-clauses for every $a, b \in V$. If $S_{3}^{\prime}$ has four $(a, b)$-clauses for any $a, b \in V$, then just as before, we can replace $S_{3}^{\prime}$ with $\perp$.

We can assume for the remainder of the proof that $S$ itself is a reduced 2-CNF that has either three, or one, or zero $(a, b)$-clauses for every $a, b \in V$. Let $a, b \in V$ be such that $S$ has exactly three $(a, b)$-clauses. By exchanging $a$ with $\neg a$ and/or $b$ with $\neg b$ we can ensure that the three $(a, b)$-clauses are $(a \vee b),(a \vee \neg b)$ and $(\neg a \vee b)$. Hence we can write $S=R \wedge(a \vee b) \wedge(a \vee \neg b) \wedge(\neg a \vee b)$, where $R$ is a reduced 2-CNF not containing any $(a, b)$-clauses. By Lemma $2(\mathrm{~b})$, we conclude that $S \sim R[\{a, b\}:=\top]$. The reduced CNF $R[\{a, b\}:=\top]$ does not have $(a, b)$-clauses. By using procedures already described in this proof, we can find a reduced 2-CNF $R^{\prime}$ equisatisfiable to $R[\{a, b\}:=\top]$ and having either three, or one, or zero $(c, d)$-clauses for every $c, d \in V$. By repeating this procedure as many times as needed, we can find a resultant reduced 2-CNF $S^{\prime}$ equisatisfiable to $S$ such that $S^{\prime}$ does not have three $(a, b)$-clauses for any $a, b \in V$. Therefore, the reduced 2-CNF $S^{\prime}$ has one or fewer $(a, b)$-clauses for every $a, b \in V$. In other words, the reduced 2-CNF $S^{\prime}$ is simple.

Throughout the remainder of this paper, when we refer to graphs, we will always mean simple graphs unless stated otherwise.

\section{5. $\mathcal{U}$ is closed under graph homeomorphism}

In this section we look at three different graph operations - subgraphing, subdivision and edge-contraction at edges not contained in triangles - and study if $\mathcal{U}$ is closed under these operations.

A graph $G$ is a subgraph of a graph $H$ if both the vertex set and edge set of $G$ are subsets of the vertex and edge sets of $H$. We will show that if a subgraph of a graph is in $\mathcal{U}$, then the graph itself must also be in $\mathcal{U}$. Edge-contraction at an edge $(u, v)$ of a graph results in a graph in which the vertices $u$ and $v$ are merged into a single new vertex $w$ and the all edges incident on $u$ or $v$ are now incident on $w$. In order to avoid the creation of multi-edges we restrict the edge-contraction operation to edges not contained in triangles. This is a necessary restriction and without it one would lose the correspondence between simple 2-CNFs and their associated simple graphs. We will show that if $G$ can be obtained from a graph in $\mathcal{U}$ via a series of edge-contractions at edges not contained in triangles, then $G$ must be in $\mathcal{U}$.

A subdivision of an edge $(u, v)$ in a graph yields a graph containing one new vertex $w$, and with an edge set replacing $(u, v)$ by two new edges $(u, w)$ and $(w, v)$. A subdivision of a graph $G$ is a graph resulting from the subdivision of edges in $G$. Two graphs are homeomorphic if they are subdivisions of the same graph. We will show that if two graphs are homeomorphic, then either both are in $\mathcal{U}$ or neither is.

A graph $G$ is a topological minor of a graph $H$ if a subdivision of $G$ is a subgraph of $H$. We will produce a complete list of graphs whose appearance as a topological minor is an obstruction to satisfiability of 2-CNFs. It is possible to embed simple graphs into $\mathbb{R}^{3}$ and to allow them to inherit the subspace topology of $\mathbb{R}^{3}$. Once embedded, homeomorphic graphs are also homeomorphic in the topological sense and topological 
graph minors are simply topological subspaces. In that sense, the ability to support unsatisfiable sentences is a topological property of graphs.

Theorem 4. If simple graphs $G$ and $H$ are homeomorphic, then $G \in \mathcal{U}$ if and only if $H \in \mathcal{U}$.

Proof. Since $G$ and $H$ are homeomorphic graphs, there exists a graph $K$ such that both $G$ and $H$ are subdivisions of $K$. It is enough to prove that $K \in \mathcal{U}$ if and only if $G \in \mathcal{U}$. In fact, it is enough to prove that $K \in \mathcal{U}$ if and only if $G^{\prime} \in \mathcal{U}$, where $G^{\prime}$ is a graph obtained via a single subdivision at an arbitrary edge $(u, v)$ in $K$. We denote by $w$ the new vertex in $G^{\prime}$ created by the subdivision.

Suppose that $K \in \mathcal{U}$. Then, there exists an unsatisfiable simple 2-CNF $S_{K}$ supported on $K$. We can write without loss of generality, $S_{K}=S \wedge(u \vee v)$, where $S$ is a simple 2-CNF not containing $(u, v)$-clauses. Note that if $S_{K}$ is not in this form, that is, if the $(u, v)$-clause is not positive in either $u$ or $v$, then we simply interchange $u$ with $\neg u$ and/or $v$ with $\neg v$ in order to bring it to the desired form. This interchange of a literal with its negation results in a different, but equisatisfiable simple 2-CNF. By abuse of notation, we will continue to refer to this new 2-CNF as $S_{K}$. We then define a simple 2-CNF $S_{G^{\prime}}$ supported on $G^{\prime}$ as $S_{G^{\prime}}=S \wedge(u \vee w) \wedge(\neg w \vee v)$. We prove that $S_{G^{\prime}}$ is unsatisfiable by showing that from any truth assignment that satisfies $S_{G^{\prime}}$, we can obtain a truth assignment satisfying $S_{K}$, leading to a contradiction.

In any truth assignment for $S_{G^{\prime}}$, either $w$ is set to $\top$ or it is set to $\perp$. If $[w:=\top]$, then it implies

$$
S_{G^{\prime}}[w:=\top]=S \wedge v \sim S[v:=\top]=S_{K}[v:=\top]
$$

is satisfiable, that is, the $2-\mathrm{CNF} S_{K}$ is satisfiable by some truth assignment which sets $[v:=\top]$. Similarly, if $[w:=\perp]$, then it implies

$$
S_{G^{\prime}}[w:=\perp]=S \wedge u \sim S[u:=\top]=S_{K}[u:=\top]
$$

is satisfiable, that is, the $2-\mathrm{CNF} S_{K}$ is satisfiable by some truth assignment that sets $[u:=\top]$. Either scenario leads to a contradiction since $S_{K}$ is assumed to be unsatisfiable. Thus, we conclude that $S_{G^{\prime}}$ is also unsatisfiable and that $G^{\prime} \in \mathcal{U}$.

Conversely, suppose that $G^{\prime} \in \mathcal{U}$. Then, there exists an unsatisfiable simple 2-CNF $S_{G^{\prime}}$, supported on $G^{\prime}$. By exchanging $u$ with $\neg u$, and/or $v$ with $\neg v$ and/or $w$ with $\neg w$, we can assume without loss of generality that either $S_{G^{\prime}}$ has the form

$$
S_{G^{\prime}}=S \wedge(u \vee w) \wedge(w \vee v) \quad \text { or } \quad S_{G^{\prime}}=S \wedge(u \vee w) \wedge(\neg w \vee v)
$$

In the first case, since $S_{G^{\prime}}$ is unsatisfiable, by setting $[w:=\top]$ we obtain an unsatisfiable 2-CNF. Thus $S_{G^{\prime}}[w:=\top]=S$ is unsatisfiable. Therefore, in this case, we have found $S \wedge(u \vee v)$, an unsatisfiable 2-CNF supported on $K$. In the other case, since $S_{G^{\prime}}$ is unsatisfiable, the 2-CNF obtained by setting $[w:=v]$ must also be unsatisfiable. Thus, $S_{G^{\prime}}[w:=v]=S \wedge(u \vee v)$ is an unsatisfiable 2-CNF supported on $K$. We conclude that $K \in \mathcal{U}$.

In view of the theorem we just proved, it is natural to attempt a classification of all members of $\mathcal{U}$ up to homeomorphism. However, we state here (without proof) that there are infinitely many graphs in $\mathcal{U}$ even up to homeomorphism. Given below is a (non-exhaustive) infinite list of graphs in $\mathcal{U}$, none of which are homeomorphic to each other.
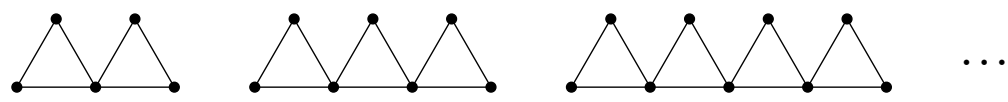

Theorem 5. Let $G$ and $H$ be simple graphs such that $G$ is a subgraph of $H$. If $G \in \mathcal{U}$, then $H \in \mathcal{U}$.

Proof. If $G \in \mathcal{U}$ then there exists an unsatisfiable simple 2-CNF $S_{G}$ supported on $G$. We denote by $S$ a simple 2-CNF supported on the graph with vertex set $V(H)$ and edge set $E(H)-E(G)$. We then define a 
simple 2-CNF $S_{H}$ as $S_{H}=S \wedge S_{G}$. The 2-CNF $S_{H}$ is supported on $H$ and is unsatisfiable since any truth assignment satisfying $S_{H}$ would also satisfy $S_{G}$. Hence $H \in \mathcal{U}$.

We note that the converse of this theorem is not true, that is, there exist graphs $G \notin \mathcal{U}$ and $H \in \mathcal{U}$ such that $G$ is a subgraph of $H$. For example, the triangle graph is not in $\mathcal{U}$ (as shown in Lemma 8) but $\triangleright \downarrow \in \mathcal{U}$ (as shown in the proof of Lemma 18).

Corollary 6. Let simple graph $G$ be a topological minor of a simple graph $H$. If $G \in \mathcal{U}$ then $H \in \mathcal{U}$.

Proof. By definition of topological minors, some subdivision $G^{\prime}$ of $G$ is isomorphic to a subgraph of $H$. By Theorem 4, if $G \in \mathcal{U}$ then $G^{\prime} \in \mathcal{U}$. By Theorem 5, if $G^{\prime} \in \mathcal{U}$ then $H \in \mathcal{U}$.

We note here that the converse of Corollary 6 is not true for the same reason that the converse of Theorem 5 is not. Corollary 6 motivates the following definition - a graph $G$ is a minimal unsatisfiability graph if both of the following conditions hold

a) $G \in \mathcal{U}$,

b) for every proper topological minor $G^{\prime}$ of $G$, we have that $G^{\prime} \notin \mathcal{U}$.

Furthermore, a set $M$ of minimal unsatisfiability graphs is complete if every graph in $\mathcal{U}$ has a subgraph that is homeomorphic to some element of $M$, that is, if every graph in $\mathcal{U}$ has an element of $M$ as a topological minor.

Since we know that minimal unsatisfiability graphs exist, we can form a set $M$ by constructing the union of all sets of minimal unsatisfiability graphs. This set is complete because each graph in $\mathcal{U}$ will have some element of $M$ as a topological minor - if not we simply add this to $M$ a minimal unsatisfiability graph that is a topological minor of this graph. Further, such a complete set must be unique because if not, then there is some minimal unsatisfiability graph $G$ in complete set $M^{\prime}$ that is not in $M$. This would result in $G$ having a proper topological minor in $\mathcal{U}$, violating the minimality of $G$. After proving the following result about the relation between edge-contraction and graphs in $\mathcal{U}$, the remainder of this paper is dedicated to finding this unique complete set of minimal unsatisfiability graphs.

Theorem 7. Let $G$ and $H$ be simple graphs such that $G$ can be obtained via a series of edge-contractions at edges of $H$ not contained in triangles. If $H \in \mathcal{U}$, then $G \in \mathcal{U}$.

Proof. If is enough to prove the theorem for the case when $G$ can be obtained from $H$ via a single edge-contraction, say at the edge $(u, v)$ not contained in any triangles in $H$. We label the new vertex in $G$ formed by the merger of $u$ and $v$ by $w$.

Suppose that $H \in \mathcal{U}$. Then, there exists an unsatisfiable simple 2-CNF $S_{H}$, supported on $H$. Without loss of generality, we can write

$$
S_{H}=S_{1} \wedge(u \vee v)
$$

where $S_{1}$ is a simple 2-CNF not containing any $(u, v)$-clauses. If $S_{H}$ is not in this form, we can exchange $u$ with $\neg u$ and/or $v$ with $\neg v$ to make the $(u, v)$-clause in $S_{H}$ positive in both variables.

One can further factor $S_{1}$ such that

$$
S_{H}=S_{2} \wedge\left(\bigwedge_{a \in A} a \vee u\right) \wedge\left(\bigwedge_{b \in B} b \vee \neg u\right) \wedge(u \vee v),
$$

where $S_{2}$ is a simple 2-CNF not containing any clauses incident on the vertex $u$, and the sets $A$ and $B$ are disjoint subsets of $(V \cup \neg V)-\{u, \neg u\}$. The sets $A$ and $B$ are disjoint because $S_{H}$ is a simple 2-CNF.

Next, we factor $S_{2}$ such that

$$
S_{H}=S_{3} \wedge\left(\bigwedge_{a \in A} a \vee u\right) \wedge\left(\bigwedge_{b \in B} b \vee \neg u\right) \wedge\left(\bigwedge_{c \in C} c \vee v\right) \wedge\left(\bigwedge_{d \in D} d \vee \neg v\right) \wedge(u \vee v),
$$


where $S_{3}$ is a simple 2-CNF not containing any clauses incident on vertices $u$ or $v$, and the sets $A, B, C, D$ are pairwise-disjoint subsets of $(V \cup \neg V)-\{u, \neg u, v, \neg v\}$. The sets $C$ and $D$ are disjoint because $S_{H}$ is a simple 2-CNF. The sets $A$ and $C$ are disjoint since the edge $(u, v)$ is not contained in a triangle. Disjointness of other pairs of sets follows similarly.

We then choose

$$
S_{G}=S \wedge\left(\bigwedge_{x \in A \cup D} x \vee w\right) \wedge\left(\bigwedge_{y \in B \cup C} y \vee \neg w\right),
$$

and note that $S_{G}$ is a simple 2-CNF supported on $G$. The pairs of sets $(A, D)$ and $(B, C)$ being disjoint implies that no clauses are lost in the process of edge-contraction. The pairs $(A, B),(A, C),(B, D)$ and $(C, D)$ being disjoint implies that $S_{G}$ is simple.

We prove that $S_{G}$ is unsatisfiable by showing that from any truth assignment that satisfies $S_{G}$, we can obtain a truth assignment satisfying $S_{H}$, leading to a contradiction.

Given a truth assignment for $S_{G}$, we can extend it to a truth assignment for $S_{H}$ by setting $[u:=w]$ and $[v:=\neg w]$. We have

$$
S_{H}[u:=w][v:=\neg w]=S \wedge\left(\bigwedge_{x \in A \cup D} x \vee w\right) \wedge\left(\bigwedge_{y \in B \cup C} y \vee \neg w\right)=S_{G},
$$

is satisfiable. This contradicts the unsatisfiability of $S_{H}$. We conclude that $S_{G}$ is unsatisfiable, and hence that $G \in \mathcal{U}$.

The converse of this theorem is not true, that is, there does exist a graph $G \in \mathcal{U}$ that can be obtained via edge-contractions of a graph $H \notin \mathcal{U}$ at edges not contained in triangles of $H$. For example, consider the graphs $G=\triangleright \downarrow$ and $H=\square$. The graph $G$ is in $\mathcal{U}$ (as shown in Lemma 18) and can be obtained from an edge-contraction from $H$. However, the graph $H$ is not in $\mathcal{U}$ (implied by Theorem 7 and Lemma 10 when combined with the fact that $H$ can be reduced to $K_{4}-e$ via other edge-contractions).

\section{Graph families that are not in $\mathcal{U}$}

Lemma 8. Let $C_{3}$ denote the triangle graph. The graph $C_{3}$ is not in $\mathcal{U}$.

Proof. We enumerate the vertex set of $C_{3}$ as $\{a, b, c\}$. Without loss of generality, every simple 2-CNF supported on $C_{3}$ can be written either in the form

$$
S=(a \vee b) \wedge(a \vee c) \wedge(b, c) \text {-clause } \quad \text { or } \quad S=(a \vee b) \wedge(\neg a \vee c) \wedge(b, c) \text {-clause. }
$$

If $S$ is not originally in this form, we modify it by interchanging $a$ with $\neg a$ and/or $b$ with $\neg b$ and/or $c$ with $\neg c$ till it is.

In the first case, setting $[a:=\top]$ gives yields a single $(b, c)$-clause. This clause can be satisfied by making the appropriate assignment for either $b$ or $c$. In the second case, by setting $[\{a, c\}:=\top]$ we get

$$
S[\{a, c\}:=\top]=(b, c) \text {-clause }[c:=\top] \sim(b, c) \text {-clause } \wedge c .
$$

The $(c)$-clause can be satisfied by setting $[c:=\top]$, while $b$ can be set to an appropriate assignment in order to satisfy the $(b, c)$-clause. Thus, the 2 -CNF $S$ is satisfiable.

Corollary 9. Let $C_{n}$ denote the cycle graph on $n$ vertices. The graph $C_{n}$ is not in $\mathcal{U}$ for any $n \geq 3$.

Proof. The graph $C_{n}$ is homeomorphic to $C_{3}$ for every $n \geq 3$. Theorem 4 and Lemma 8 therefore imply that $C_{n} \notin \mathcal{U}$. 
Lemma 10. Let $K_{4}$ denote the complete graph on four vertices. Let $K_{4}-e$ denote the graph obtained by deleting a single edge e from $K_{4}$. The graph $K_{4}-e$ is not in $\mathcal{U}$.

Proof. We enumerate the vertex set of $K_{4}$ as $\{a, b, c, d\}$. Let $e$ denote the edge $(c, d) \in E\left(K_{4}\right)$ such that $(c, d) \notin E\left(K_{4}-e\right)$. Every 2-CNF $S$ supported on $K_{4}-e$ can be written either in the form

$$
\begin{aligned}
& S=(a \vee b) \wedge(a \vee c) \wedge(a \vee d) \wedge(b, c) \text {-clause } \wedge(b, d) \text {-clause, or } \\
& S=(a \vee b) \wedge(a \vee c) \wedge(\neg a \vee d) \wedge(b, c) \text {-clause } \wedge(b, d) \text {-clause. }
\end{aligned}
$$

If $S$ is not already in the desired form, then we can interchange each variable with its negation till it is.

In the first case, by setting $[a:=\top]$ we obtain

$$
S[a:=\top]=(b, c) \text {-clause } \wedge(b, d) \text {-clause. }
$$

This can be satisfied by making appropriate assignments for $c$ and $d$ so that they satisfy each of the clauses. In the second case, we can set $[a:=\top]$ to obtain

$$
S[a:=\top]=d \wedge(b, c) \text {-clause } \wedge(b, d) \text {-clause. }
$$

This resulting CNF can be satisfied by setting $[d:=\top]$, by choosing an assignment for $b$ that would satisfy the $(b, d)$-clause and by then choosing an assignment for $c$ that would satisfy the $(b, c)$-clause. We conclude that $S$ is always satisfiable, and therefore, the graph $K_{4}-e$ is not in $\mathcal{U}$.

Lemma 11. Tree graphs are not in $\mathcal{U}$.

Proof. Every tree graph can be reduced via edge-contractions to a single-vertex graph. A single-vertex graph is clearly not in $\mathcal{U}$. The result follows from Theorem 7 .

\section{Structure of graphs with two or three independent cycles}

In this section we prove four lemmas about the structure of graphs that have either two or three independent cycles. These structural results are needed for proving the results in Section 8, including the main result of this paper (Theorem 20).

Lemma 12. Every connected simple graph having exactly two copies of $C_{3}$ as subgraphs has one of the following three graphs as a topological minor.
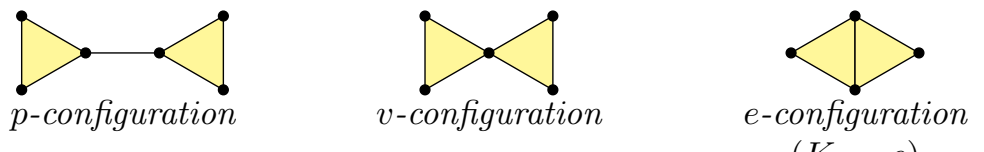

$\left(K_{4}-e\right)$

Remark 13. For convenience, we label the three graphs as the p-configuration ( $p$ stands for path), the $v$-configuration ( $v$ stands for vertex) and e-configuration (e stands for edge) respectively. The two copies of $C_{3}$ have been filled in for easy visual identification.

Proof. We construct this list of topological minors from the bottom up. Two copies of $C_{3}$ can be put together to create a connected simple graph in exactly three ways

a) either the two copies of $C_{3}$ share zero vertices,

b) or they share exactly one vertex,

c) or they share exactly two vertices (that is, they share an edge). 
In the first case, since the graph is still supposed to be connected, we claim that the two copies of $C_{3}$ must be connected by one or more paths. Such a graph will always have the two copies connected by a single path as a subgraph. Hence, the graph will also always have the $p$-configuration as a topological minor.

In the second case, the graph will always have the $v$-configuration as a subgraph and therefore, also as a topological minor. Similarly, in the third case, the graph will always have the $e$-configuration as a subgraph and therefore, also as as topological minor.

Lemma 14. Every connected simple graph having exactly two or more independent cycles has one of the three graphs in Lemma 12 as a topological minor.

Proof. Let $G$ be a connected simple graph with two or more independent cycles. If any two cycles share one or more edges, then $K_{4}-e$ is a topological minor of $G$. If no pair of cycles share any edges, but at least one pair shares a vertex, then the $v$-configuration is a topological minor of $G$. If every pair of cycles share neither any edges nor any vertices, then using the connectedness of $G$, we infer that there must be a path connecting vertices in every pair of cycles. Thus, in this case, the $p$-configuration is a topological minor of $G$.

Lemma 15. Every connected simple graph having three or more copies of $C_{3}$ as subgraphs has one of the following 15 graphs as a topological minor.
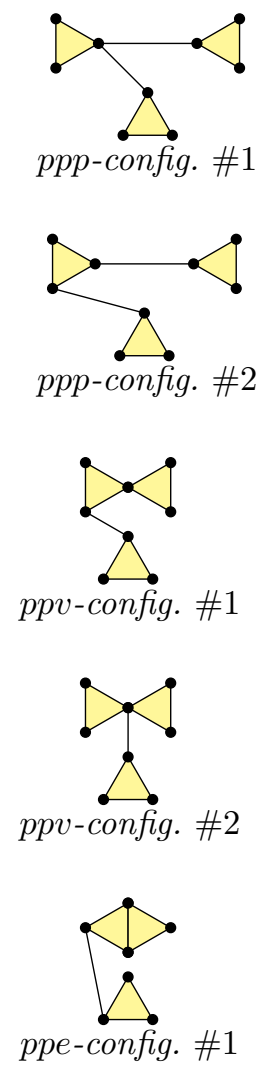
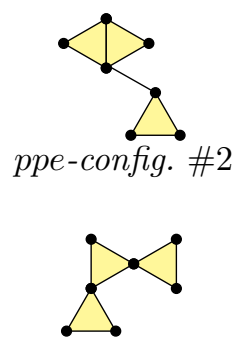

pvv-configuration

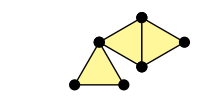

pve-configuration

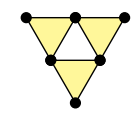

vvv-config. \#1

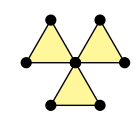

vvv-config. \#2

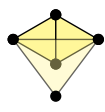

vve-config. \#1

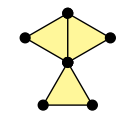

vve-config. \#2

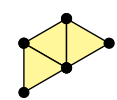

vee-configuration

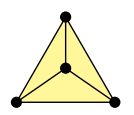

eee-config. \#1

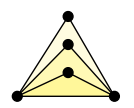

eee-config. \#2

Remark 16. Labels for each of the 15 graphs (like ppp-config. \#1) are explained as part of the proof. The three copies of $C_{3}$ that we use for this labeling have been filled in for the sake of easy visual identification.

Proof. We arrive at this list of topological minors by constructing them from the bottom up. From the proof of Lemma 12, we know that every pair of $C_{3}$ can be joined in exactly one of three ways and that these three ways are inequivalent when considering the topological minor relation, that is, no graph in Lemma 12 
is a topological minor of another graph in Lemma 12. Graphs with three or more copies of $C_{3}$ will have at least $\left(\begin{array}{l}3 \\ 2\end{array}\right)=3$ distinct pairs of $C_{3}$.

To enumerate all possible joinings of these three pairs of $C_{3}$, we form all three-letter words formed using the letters $\{p, v, e\}$, with repetition allowed, but order being irrelevant. For example, the word ppe would correspond to the family of graphs where the two pairs of $C_{3}$ are joined via the $p$-configuration, while the third pair of $C_{3}$ is joined via the $e$-configuration. We also note that some words correspond to multiple non-isomorphic configurations. We analyze each case separately below

a) $p p p$-configuration. After joining the first pair of $C_{3}$ in a $p$-configuration, the third copy of $C_{3}$ can be added in two different non-isomorphic ways (such that the first and third, as well as the second and third are joined via the $p$-configuration). Thus, we obtain only two configurations in this case - ppp-config. \#1 and $p p p$-config. \#2.

b) $p p v$-configuration. After joining the first pair of $C_{3}$ in a $v$-configuration, the third copy of $C_{3}$ can be added in two non-isomorphic ways. Thus, we obtain only two configurations in this case, namely ppv-config. \#1 and $p p v$-config. \#2.

c) ppe-configuration. After joining the first pair of $C_{3}$ in an $e$-configuration, the third copy of $C_{3}$ can be added in two non-isomorphic ways. Thus, we obtain only two configurations in this case, namely ppe-config. \#1 and ppe-config. \#2.

d) $p v v$-configuration. After joining the first pair of $C_{3}$ in a $v$-configuration, the third copy of $C_{3}$ can be added in only one way. Thus, we obtain only one configurations in this case, namely the $p v v$-configuration.

e) pve-configuration. After joining the first pair of $C_{3}$ in an $e$-configuration, the third copy of $C_{3}$ can added in only one way. Thus, we obtain only one configurations in this case, namely the pve-configuration.

f) pee-configuration. After joining the first pair of $C_{3}$ in an $e$-configuration, the third copy of $C_{3}$ cannot be added in a way that it is shares an edge with the first and is connected by a path to the second. Thus, we do not obtain configurations in this case.

g) $v v v$-configuration. After joining the first pair of $C_{3}$ in an $v$-configuration, the third copy of $C_{3}$ can be added in two non-isomorphic ways. Thus, we obtain only two configurations in this case, namely vvv-config. \#1 and vvv-config. \#2.

h) vve-configuration. After joining the first pair of $C_{3}$ in an $e$-configuration, the third copy of $C_{3}$ can be added in two non-isomorphic ways. Thus, we obtain only two configurations in this case, namely vve-config. \#1 and vve-config. \#2.

i) vee-configuration. After joining the first pair of $C_{3}$ in an $e$-configuration, the third copy of $C_{3}$ can added in only one way. Thus, we obtain only one configurations in this case, namely the vee-configuration.

j) eee-configuration. After joining the first pair of $C_{3}$ in an $e$-configuration, the third copy of $C_{3}$ can be added in two non-isomorphic ways. Thus, we obtain only two configurations in this case, namely eee-config. \#1 and eee-config. \#2.

Lemma 17. Every connected simple graph having three or more independent cycles has one of the following four graphs as a topological minor.
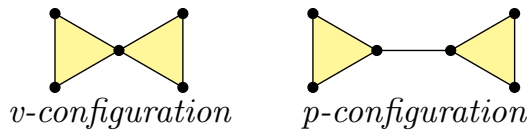

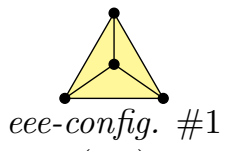

$\left(K_{4}\right)$

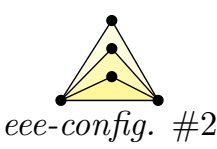

$\left(K_{1,1,3}\right)$

Proof. It is enough to show that every graph listed in Lemma 15 has one of the four graphs listed above as a topological minor. The $p$-config. is a subgraph of both the $p p p$-configs., both the $p p v$-configs., both ppe-configs., the pvv-config., and the pve-config. Of the remaining configurations, both the $v v v$-configs., both the vve-configs., and the vee-config. contain the $v$-config. as a subgraph.

The first eee-configuration is isomorphic to $K_{4}$ while the second is isomorphic to $K_{1,1,3}$. Hence, all 15 configurations have at least one of the four graphs listed above as a topological minor. 


\section{The complete set of minimal unsatisfiability graphs}

We note that a simple graph $G$ is in $\mathcal{U}$ if and only if some connected component of $G$ is in $\mathcal{U}$. We proceed to make some more observations about the graph-family $\mathcal{U}$.

Lemma 18. The four graphs in Lemma 17 are minimal unsatisfiability graphs.

Proof. Consider the following unsatisfiable 2-CNFs.

$$
\begin{aligned}
& S_{1}=(a \vee b) \wedge(\neg a \vee c) \wedge(\neg b \vee c) \wedge(\neg c \vee d) \wedge(\neg c \vee e) \wedge(\neg d \vee \neg e), \\
& S_{2}=(a \vee b) \wedge(\neg a \vee c) \wedge(\neg b \vee c) \wedge(\neg c \vee d) \wedge(\neg d \vee e) \wedge(\neg d \vee f) \wedge(\neg e \vee \neg f), \\
& S_{3}=(a \vee b) \wedge(a \vee c) \wedge(\neg a \vee d) \wedge(\neg b \vee \neg c) \wedge(b \vee \neg d) \wedge(c \vee \neg d) \text { and } \\
& S_{4}=(a \vee b) \wedge(\neg a \vee d) \wedge(b \vee c) \wedge(\neg b \vee d) \wedge(\neg b \vee e) \wedge(\neg c \vee \neg d) \wedge(\neg d \vee \neg e) .
\end{aligned}
$$

Their associated graphs are the four graphs listed above in order. Since these 2-CNFs are unsatisfiable, the graphs are in $\mathcal{U}$. To prove that they are minimal unsatisfiability graphs, we need to show that none of the proper topological minors of these graphs are in $\mathcal{U}$. Since the four listed graphs are not subdivisions of other graphs, it is enough to show that every proper subgraph of these graphs is not in $\mathcal{U}$.

Every proper subgraph of the $v$-configuration is either a subgraph of $\perp<$ or $D$. Both of these graphs can be reduced via edge-contractions to $C_{3}$. In Lemma 8 we proved $C_{3} \notin \mathcal{U}$ and hence by Theorem 7 we can conclude that neither $\downarrow<$ nor $\triangleright$ ! is in $\mathcal{U}$. Therefore, by Theorem 5, none of the subgraphs of $v$-configuration are in $\mathcal{U}$.

Every proper subgraph of the $p$-configuration is either a subgraph of $\downarrow \neg$. $><$ or $\triangleright \backslash$. The first two of these graphs can be reduced via edge-contractions to proper subgraphs of the $v$-configuration. Since we proved that none of the proper subgraphs of the $v$-configuration are in $\mathcal{U}$, by Theorem 7 we can conclude that $\triangleright \neg$ nor $\triangleright<$ is in $\mathcal{U}$. Lastly, the graph $\triangleright \downarrow$ is in $\mathcal{U}$ if and only if at least one of its connected components is in $\mathcal{U}$. In light of Lemma 8, we conclude that $\triangleright \downarrow \downarrow$ is not in $\mathcal{U}$. Theorem 5 implies that none of the proper subgraphs of the $p$-configuration are in $\mathcal{U}$.

In Lemma 10, we proved that $K_{4}-e \notin \mathcal{U}$. Since every proper subgraph of $K_{4}$ is also a subgraph of $K_{4}-e$, using Theorem 5 we conclude that none of the proper subgraphs of $K_{4}$ are in $\mathcal{U}$.

Every proper subgraph of $K_{1,1,3}$ is either a subgraph of or $K_{2,3}($ ) , both of which can be reduced via edge-contractions to $K_{4}-e$. In Lemma 10 we proved $K_{4}-e \notin \mathcal{U}$ and hence by Theorem 7 we conclude that none of the proper subgraphs of $K_{1,1,3}$ are in $\mathcal{U}$.

Corollary 19. Every connected simple graph having three or more independent cycles is in $\mathcal{U}$.

Proof. From Lemma 17 we know that every connected simple graph having three or more independent cycles has one of the four graphs listed in that lemma as a topological minor. Since we proved in Lemma 18 that all four of these graphs are in $\mathcal{U}$, the result follows from Corollary 6.

Theorem 20. The set $\{\triangleright \downarrow, \triangleright \triangleleft, \mathbb{A}\}$ is the complete set of minimal unsatisfiability graphs.

Proof. We denote the set by $M$. Since we proved in Lemma 18 that the elements of $M$ are minimal unsatisfiability graphs, it suffices to show that a simple graph $G$ is in $\mathcal{U}$ if and only if $G$ has some element of $M$ as a topological minor. Corollary 6 implies the "if" part of this statement. We now show that every graph $G \in \mathcal{U}$ has some element of $M$ as a topological minor. Throughout the remainder of the proof we suppose that $G \in \mathcal{U}$.

If $G$ is connected and has three or more independent cycles, then the result follows from Lemma 17. If $G$ is connected and has exactly two independent cycles, then by Lemma 14 it follows that either $G$ has an element of $M$ as a topological minor or $G$ has $K_{4}-e$ as a topological minor. For the latter case, we note that a graph with exactly two independent cycles having $K_{4}-e$ as a topological minor must in fact be a graph 
with two cycles sharing one or more edges along with zero or more leaf edges (edges incident on vertices of degree one). Such a graph can be reduced via edge-contractions to $K_{4}-e$. Using Theorem 7 , we conclude that $K_{4}-e \in \mathcal{U}$. However, this contradicts the result proved in Lemma 10. We therefore conclude that any connected graph $G$ having exactly two independent cycles must have some element of $M$ as a topological minor.

If $G$ is connected and has exactly one cycle, then $G$ can be reduced to $C_{3}$ by edge-contractions. We know from Lemma 8 that $C_{3} \notin \mathcal{U}$. Thus, Theorem 7 implies $G \notin \mathcal{U}$, which is a contradiction. Thus $G$ cannot have exactly one cycle. If $G$ is connected and has no cycles, then $G$ is a tree graph. We know from Lemma 11 that tree graphs are not in $\mathcal{U}$, which is a contradiction. Thus $G$ cannot have zero cycles.

Finally, suppose $G \in \mathcal{U}$ and $G$ is not connected. Then, some connected component $G^{\prime}$ of $G$ is in $\mathcal{U}$. By our previous argument, we know that $G^{\prime}$ has some element of $M$ as a topological minor. This element of $M$ must therefore also be a topological minor of $G$. We have showed that any graph in $\mathcal{U}$, connected or not, has an element of $M$ as a topological minor.

Corollary 21. A simple graph $G$ can support an unsatisfiable 2-CNF if and only if one of the four graphs in Lemma 17 is a topological minor of $G$.

Proof. This is simply an alternative way of stating the result of Theorem 20 using the full definition of $\mathcal{U}$ and of the complete set of minimal unsatisfiability graphs.

Remark 22. As embedded graphs in $\mathbb{R}^{3}$, the four graphs in Theorem 20 are homeomorphic to one of the following 1-dimensional cell complexes
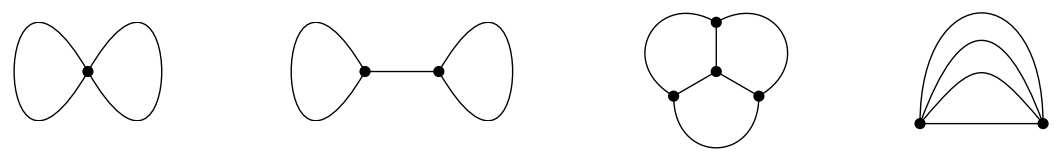

One can immediately identify unsatisfiable sentences supported on these cell complexes. For the first one, this is the (unreduced) 2-CNF $(x \vee x) \wedge(\neg x \vee \neg x)$. For the second cell complex, the (unreduced) 2-CNF $(x \vee x) \wedge(\neg x \vee \neg y) \wedge(y \vee y)$ is unsatisfiable. For the third, the cell complex is the same as $K_{4}$ and any unsatisfiable 2-CNF supported on $K_{4}$ will suffice. For the fourth cell complex, the (unreduced) 2-CNF $(x \vee y) \wedge(\neg x \vee y) \wedge(x \vee \neg y) \wedge(\neg x \vee \neg y)$ is unsatisfiable.

\section{Conclusion - relation between our result and Robertson-Seymour theorem}

In our main result, Theorem 20, we showed that given a simple graph, all reduced sentences supported on it will be satisfiable if and only if four specific graphs are forbidden as topological minors of the original graph. Thus it is natural to ask if the forbidden minors theory of Robertson-Seymour [9]-[10] applies to our problem.

\subsection{Graph minors, pseudo-minors and topological minors}

A graph $G$ is traditionally said to be a minor of $H$ if it can be obtained from $H$ by a series of edge-deletions, vertex-deletions and/or edge-contractions. The edge-contraction operation involves picking an edge in the graph, deleting it, then merging the two end-vertices of that edge. The problem is that this merging can potentially create multi-edges (meaning, even if $H$ is a simple graph, its minor $G$ might be a multigraph). There are three ways to deal with this creation of multigraphs in our discussion:

a) We can allow it: in that case, we cannot restrict ourselves to only dealing with simple graphs and we will have to consider sentences supported on multigraphs too. This can be done but is complicated and we consider it to be beyond the scope of this paper.

b) We can rectify it: in this case, we can let the multi-edge be created, to only then "throw out" the extra edge. However, the trouble here is this "throwing out" operation. Since we have for each 2-CNF an associated multigraph, throwing out this extra edge corresponds to throwing out a clause from the 2-CNF. There is no real justification for doing this. Seen another way, throwing out an extra edge from the graph without justification will break the correspondence that the graph has with the 2-CNF it supports. 
c) We can forbid it: and this is the path we choose in this paper. To forbid the creation of multigraphs we allow edge-contractions but only at edges not contained in triangles.

Hence the relation we consider is not that of a "minor" but that of a "pseudo-minor" (edge-contractions are allowed but only at edges not contained in triangles). We note here that pseudo-minor is not a standard term used among graph theorists.

\subsection{Robertson-Seymour graph minor theorem}

The Robertson-Seymour graph Theorem (RST) states that if a graph-family $\mathcal{F}$ is minor-closed, meaning that every minor of a graph in $\mathcal{F}$ is also in $\mathcal{F}$, then there are at most finitely-many forbidden minors of $\mathcal{F}^{c}$ - meaning a graph belongs to $\mathcal{F}$ if and only if it does not contain as a minor any of these forbidden graphs. In our case, a suitable candidate for $\mathcal{F}$ is the set of graphs that support only satisfiable 2-CNFs. According to our notation, this is $\mathcal{U}^{c}$ ). If $\mathcal{U}^{c}$ is shown to be minor-closed then by RT we will be able to find a finite number of forbidden minors. However, as discussed above, we cannot meaningfully talk about minors of graphs in the context of 2-CNFs while restricting ourselves to simple graphs only. We deal with the relation of a "pseudo-minor" and hence RST does not apply. Unfortunately, the authors of this paper are not aware of a Robertson-Seymour-type theorem for pseudo-minors.

To summarize, if $\mathcal{U}^{c}$ were shown to be minor-closed, then RST would imply finitely-many forbidden minors. In this paper we have established that $\mathcal{U}^{c}$ is pseudo-minor-closed. However, RST does not apply so it appears as though it is possible that there might be infinitely-many forbidden pseudo-minors. Fortunately, our result implies that are in fact only finitely many.

We have also shown that the graph family $\mathcal{U}^{c}$ is topological-minor-closed. However, the topological minor relation is not a well-quasi-ordering on the set of finite graphs and therefore, RST does not apply to it. Fortunately, our main theorem established that $\mathcal{U}^{c}$ has exactly four forbidden topological minors (we call this forbidden set the complete set of minimal unsatisfiability graphs).

\subsection{Computational complexity}

There are subtle results involving the computational complexity implications of our result as well. Since 2-SAT is known to be in complexity class P, it would at first glance appear that we have made the problem worse: one can infer from our main theorem that the satisfiability of a given 2-CNF can be established by looking for four specific topological minors in its associated graphs. If these four topological minors are absent, then the 2-CNF must be satisfiable. If they are present, then the 2-CNF may or may not be satisfiable. This may appear to be setback since the GRAPH MINOR decision problem is known to be NP-complete, implying that the GRAPH TOPOLOGICAL MINOR problem will also be NP-complete.

However, on closer inspection, one realizes that the decision problem of determining if a fixed graph is present as a topological minor can actually be resolved in polynomial time as a consequence of the graph minor theorem. Since our set of forbidden topological minors is finite we can therefore guarantee that the task of searching for them can be accomplished in polynomial time.

Thus, we have done "no worse" than the original 2-SAT problem as both can still be solved in polynomial time. We emphasize however that as we have stated earlier, our goal in this paper is the characterization of unsatisfiable reduced 2-CNFs as opposed to questions of algorithmic efficiency for solving the satisfiability problem for 2-CNFs for which, in any case, efficient algorithms already exist. We hope that the techniques developed in this paper when generalized to hypergraphs might shed light on the structure of unsatisfiable 3-CNFs.

Acknowledgement:. The authors thank Yuliy Baryshnikov for suggesting the problem of studying satisfiability from the viewpoint of the underlying structure of the sentences and for early discussions on the subject. 


\section{References}

[1] M. R. Krom, The decision problem for a class of first-order formulas in which all disjunctions are binary, Mathematical Logic Quarterly 13 (1-2) (1967) 15-20. arXiv:https://onlinelibrary.wiley.com/doi/pdf/10.1002/malq.19670130104, doi:10.1002/malq.19670130104.

URL https://onlinelibrary.wiley.com/doi/abs/10.1002/malq.19670130104

[2] S. Even, A. Itai, A. Shamir, On the complexity of timetable and multicommodity flow problems, SIAM Journal on Computing 5 (4) (1976) 691-703. arXiv:https://doi.org/10.1137/0205048, doi:10.1137/0205048 URL https://doi.org/10.1137/0205048

[3] B. Aspvall, M. F. Plass, R. E. Tarjan, A linear-time algorithm for testing the truth of certain quantified boolean formulas, Information Processing Letters 8 (3) (1979) 121 - 123. doi:https://doi.org/10.1016/0020-0190 (79) 90002-4.

URL http://www.sciencedirect.com/science/article/pii/0020019079900024

[4] T. Feder, Network flow and 2-satisfiability, Algorithmica 11 (3) (1994) 291-319. doi:10.1007/BF01240738. URL https://doi.org/10.1007/BF01240738

[5] S. A. Cook, The complexity of theorem-proving procedures, in: Proceedings of the Third Annual ACM Symposium on Theory of Computing, STOC '71, ACM, New York, NY, USA, 1971, pp. 151-158. doi:10.1145/800157.805047. URL http://doi.acm.org/10.1145/800157.805047

[6] L. A. Levin, Universal enumeration problems, Problemy Peredači Informacii 9 (3) (1973) 115-116.

[7] T. J. Schaefer, The complexity of satisfiability problems, in: Proceedings of the Tenth Annual ACM Symposium on Theory of Computing, STOC '78, ACM, New York, NY, USA, 1978, pp. 216-226. doi:10.1145/800133.804350. URL http://doi.acm.org/10.1145/800133.804350

[8] R. M. Karp, Reducibility among combinatorial problems, in: Complexity of computer computations, Springer, 1972, pp. $85-103$.

[9] N. Robertson, P. Seymour, Graph minors I. Excluding a forest, Journal of Combinatorial Theory, Series B 35 (1) (1983) 39 - 61. doi:https://doi.org/10.1016/0095-8956(83)90079-5. URL http://www.sciencedirect.com/science/article/pii/0095895683900795

[10] N. Robertson, P. Seymour, Graph minors XX. Wagner's conjecture, Journal of Combinatorial Theory, Series B 92 (2) (2004) 325 - 357, special Issue Dedicated to Professor W.T. Tutte. doi:https://doi.org/10.1016/j.jctb.2004.08.001. URL http://www.sciencedirect.com/science/article/pii/S0095895604000784 